\title{
Commentary: Obesity and atrial fibrillation after cardiac surgery: The weight continues
}

\author{
Kimberly A. Holst, MD
}

\author{
From the Department of Cardiovascular Surgery, Mayo Clinic, Rochester, Minn. \\ Disclosures: Author has nothing to disclose with regard to commercial support. \\ Received for publication April 15, 2019; accepted for publication April 16, 2019; available ahead of print June 5, \\ 2019. \\ Address for reprints: Kimberly A. Holst, MD, Department of Cardiovascular Surgery, Mayo Clinic, 200 First St \\ SW, Rochester, MN 55905 (E-mail: holst.kimberly1@mayo.edu). \\ J Thorac Cardiovasc Surg 2020;159:941-2 \\ $0022-5223 / \$ 36.00$ \\ Copyright (C) 2019 by The American Association for Thoracic Surgery \\ https://doi.org/10.1016/j.jtcvs.2019.04.053
}

In this issue of the Journal, Serban and coauthors ${ }^{1}$ outline their series of de novo postoperative atrial fibrillation (PoAF) in 156 patients after elective cardiac surgery and found that obese patients had an increased rate and burden of PoAF, with a median of 5 episodes versus 4 episodes in nonobese patients. Serban and coauthors ${ }^{1}$ are to be commended on their diligence in electrophysiologic monitoring and assessment, including manual review of continuous monitoring and detailed analysis of incidence, burden, and timing of events of atrial fibrillation after elective cardiac surgery in their study population. The question addressed in the article is whether there is an increased burden of PoAF in obese patients; the answer is yes. Questions left for the reader, however, are numerous. We are left questioning the clinical significance of this difference, the effects of other factors on PoAF burden, and, most importantly, what to do about it.

The association between obesity and atrial fibrillation is not new within the field of cardiac surgery, or outside cardiac surgery, 2,3 and the relationships between atrial fibrillation and clinical outcomes are well described. ${ }^{4}$ What Serban and coauthors ${ }^{1}$ do not explore, and we still do not understand, is the multitude of other factors that may also affect the association between obesity and PoAF incidence and duration. Were the intraoperative and postoperative courses different for obese patients? That is, were procedure times longer, were pump times longer, did they require more fluid, was their volume status on postoperative day 3 (during the peak incidence of de novo PoAF) different? Was there an association of PoAF with body mass index or potentially with other metabolic factors, presumably more common in obese patients? What is the impact of obstructive sleep apnea? ${ }^{5}$ Although the mechanism connecting PoAF and obesity is outside the scope of this article, consideration of these factors would have been informative, particularly because obese patients tended to have episodes of PoAF on the third

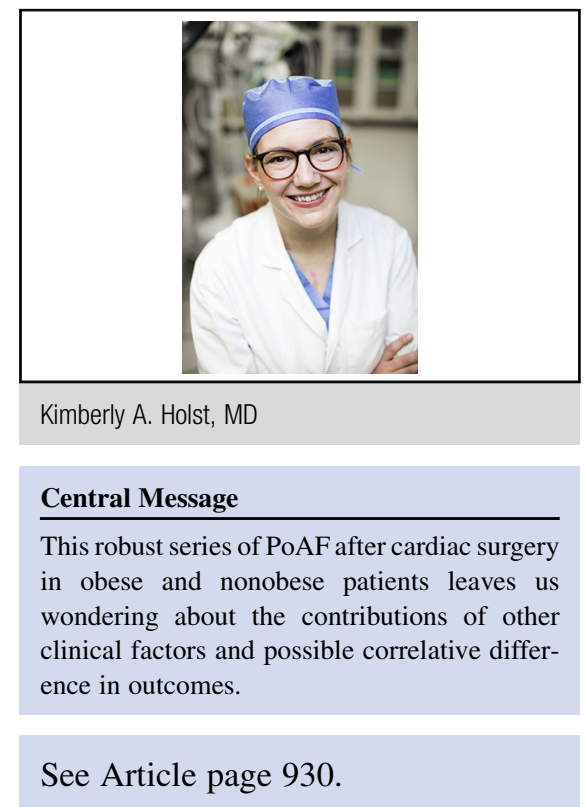

post-operative day, compared with the second day for nonobese patients.

We are also left to wonder about the clinical impact of the relative increase in atrial fibrillation in obese patients and nonobese patients. Was there a difference in length of hospital stay? A difference in stroke rate? A difference in late freedom from atrial fibrillation?

In conclusion, the article of Serban and coauthors ${ }^{1}$ contains a multitude of data regarding the incidence and burden of PoAF in their population of patients after elective cardiac surgery. Although the information regarding PoAF is robust, and there is an association between body mass index of at least $30 \mathrm{~kg} / \mathrm{m}^{2}$ and increased burden of PoAF, there is a relative lack of information available regarding other clinical factors and outcomes. In the end, we must maintain a high index of suspicion for atrial fibrillation in both obese and nonobese patients and "weight" for future studies to delineate how additional clinical and surgical factors influence the difference in PoAF burden in these patients.

\section{References}

1. Serban C, Arinze JT, Starreveld R, Lanters EAH, Yaksh A, Kik C, et al. The impact of obesity on early postoperative atrial fibrillation burden. J Thorac Cardiovasc Surg. 2020;159:930-8.e2.

2. Perrier S, Meyer N, Hoang Minh T, Announe T, Bentz J, Billaud P, et al. Predictors of atrial fibrillation after coronary artery bypass grafting: a Bayesian analysis. Ann Thorac Surg. 2017;103:92-7. 
3. Lavie CJ, Pandey A, Lau DH, Alpert MA, Sanders P. Obesity and atrial fibrillation prevalence, pathogenesis, and prognosis: effects of weight loss and exercise. J Am Coll Cardiol. 2017;70:2022-35.

4. Rankin JS, Lerner DJ, Braid-Forbes MJ, Ferguson MA, Badhwar V. One-year mortality and costs associated with surgical ablation for atrial fibrillation concomitant to coronary artery bypass grafting. Eur J Cardiothorac Surg 2017;52:471-7.

5. Feng TR, White RS, Ma X, Askin G, Pryor KO. The effect of obstructive sleep apnea on readmissions and atrial fibrillation after cardiac surgery. $J$ Clin Anesth $2019 ; 56: 17-23$ 